\title{
Dental Caries Prevalence and Untreated Caries among Children in a School-Based Dental Health Program Using dft/DFT and Significant Caries Index (SCI)
}

\author{
Tarik Shounia ${ }^{1}$,Salwa Atwan ${ }^{2 *}$ and Divesh Byrappagari ${ }^{3}$ \\ ${ }^{1}$ Associate Professor, University of Detroit Mercy, School of Dentistry/Pediatric Section, Detroit, Michigan, USA \\ ${ }^{2}$ Associate professor, Chief of Pediatric Services, University of Detroit Mercy, Detroit, MI, USA \\ ${ }^{3}$ Associate Professor/Office of Dental Public Health and Outreach, University of Detroit Mercy, Detroit, Michigan, USA \\ *Corresponding Author: Salwa Atwan, Chief of Pediatric Services, University of Detroit Mercy, Detroit, MI, USA.
}

Received: July 22, 2019; Published: September 13, 2019

DOI: $10.31080 /$ ASDS.2019.03.0639

\begin{abstract}
Background: Significant Caries Index (SCI) was proposed by WHO to draw attention to those individuals with the highest caries score in each population. The aim of the study was to evaluate the prevalence of dental caries and untreated caries, using combined decay, filling for primary teeth (dft) and permanent teeth (DFT) and SCI indices.

Methods: 144 School children aged 4-13 years old were examined in a mobile dental clinic as part of the school-based dental services program. Combined dft/DFT and Significant Caries Index were used to evaluate dental caries prevalence. Dental caries were recorded when cavitation was observed clinically or radiographically. The services provided to children included comprehensive diagnostic, preventive and restorative treatment.

Results: The mean combined dft/DFT for all groups was 3.18. As the age increased, the mean decreased with no significant differences. The SCI for all age groups was significantly higher than combined dft/DFT. The caries prevalence for this school was 63 . 9\%. About $77 \%$ of children had untreated caries.

Conclusion: The study reported high prevalence of caries experience and untreated caries. The SCI is designed to focus attention to high caries risk group; it is useful indicator and helps in targeting the preventive program for this group. IRB \# $1819-49$.
\end{abstract}

Keywords: Dental Caries; Dft/DFT; Significant Caries Index (Sci)

\section{Background}

Dental caries is the most common childhood disease worldwide. Between 60 and $90 \%$ of children are affected, but the majority of dental decay remain untreated [1]. Childhood dental caries has been reported to be the most prevalent infectious disease in our nation. It is 5 times more common than asthma and 7 times more common than hay fever among 5 to 17 year old American children [2]. In 1996, oral disease resulted in 1.6 million days of school lost; an average of 3.1 day per 100 students in the United States [3]. In 2009 - 2010, the prevalence of untreated decay and decay experience among 6 - 8 year old children examined by National Health and Nutrition Examination Survey (NHANES) throughout the United States was $17 \%$ and $45 \%$ respectively [4].

Data from National Health and Nutrition Examination survey for 2011 - 2012 reported that approximately $37 \%$ of children aged 2 - 8 years in the US had experienced dental caries, and $14 \%$ of the same age group had untreated tooth decay in primary teeth. While $21 \%$ of children aged 6 - 11 had experienced dental caries in permanent teeth and $6 \%$ had untreated decay in permanent teeth [5]. 
Data from Michigan Department of Health and Human Services reported that over half of all third grade children age 6 - 8 years had experienced dental caries in primary or permanent teeth. Twenty five percent have untreated dental decay, and $28 \%$ of children bear $75 \%$ of teeth affected by dental caries. Children with untreated dental caries average 2.3 teeth in primary teeth and 1.5 teeth in permanent teeth [6].

Many studies in developed countries show remarkably improvement in child dental health, but significant proportion of low income, socially vulnerable and minority children continue at high risk of dental caries [3,7-9].

Based on these findings, a new index called "Significant Caries Index" (SCI) was proposed by WHO to draw attention to those individuals with the highest caries score in each populations [10]. Expressing caries prevalence as the mean DMFT/dmft value does not correctly reflect the skewed distribution, leaving high caries groups undiscovered in the population.

One in four American children was born into poverty suffering twice as much tooth decay as their more affluent peers and more likely having no access to oral health care. Therefore, dental disease in children in the US remains a major public health problem.

Access to oral health care is a problem for many children and families. Many factors affected accesses to dental care e.g. lack of parent awareness of oral health needs of their children, parent often are caution about taking time from work to seek health care for their children, lacking dental insurance, transportation. Therefore brining health services to the school is an efficient way to reach the children that are in most need to dental treatment and preventive services.

In September 2015, as a part of the University of Detroit Mercy School of Dentistry (UDMSOD) Titans for Teeth Mobile Clinic Program (TFTMC), senior students and faculty members traveled to schools throughout Metropolitan Detroit area to provide comprehensive diagnostic, preventive and restorative treatment to hundreds of school aged children. TFTMC is fully equipped mobile program with eight chairs clinic; four chairs are inside of the van and four chairs are portable units set-up on site (the school). The aim of the study was to evaluate the prevalence of dental caries and untreated caries in primary and permanent teeth using DFT/ $\mathrm{dft}$, and SCI in a group of school children aged 4 - 13 years who participated in the TFTMC program. In addition to providing access to dental services for underserved children, the program also increases the awareness of future dental providers to the needs of the community services.

\section{Methods}

Participants: Starting September 2015, TFTMC provided dental services to many schools in the City of Detroit. Data for this study were collected from one of these schools. Children in this school were African American and enrolled in the Free \& Reduced Lunch (FRL) program, which is available to children from low income families that attend the school. A majority of the children at the school were from low-income families based on the FRL participation. Visitation permission and coordination with the school was obtained from school principal to arrange days for collecting data and treatment. All children were required to have a signed consent form completed by their parent or legal guardians prior to services. One hundred seventy three consent forms were returned. A complete dental examination with radiograph was conducted for 144 children. There were 68 (47.2\%) males and 76 (52.8\%) females. The sample was divided into four age groups 4 - 5 , 6 - 8, 9 - 11 and 12 - 13 year old. Instruments: Oral examination was carried out using mouth mirror and explorer. Bitewing radiographs were taken to detect proximal caries. Procedures: Dental caries recorded clinically at cavitation level and radiographically at D1 level. DMFT, dmft, SCI indices were used to evaluate dental caries prevalence. Due to difficulty in distinguishing between extracted primary teeth for caries or exfoliation, the missing teeth were not recorded. Therefore, combined dft and DFT were used as a tool to record dental caries status for the sample.

The SCI index was calculated as follows: individual were sorted according to their sum of $\mathrm{dft}$ and DFT values. The one third of the population with highest caries score is selected and the mean for this subgroup is calculated.

Treatment is performed by dental senior students under supervision of licensed dental and dental hygiene faculty. The services provided are dental examination, prophylaxis, oral health instructions, radiographs, sealants, restorative care, extractions, referrals and emergency services. Complex treatment as root canal for permanent teeth, space maintainer, and treatment under sedation were referred to the dental school or Children Hospital of Michigan. 
Data Analysis: The statistical analysis was conducted using IBM SPSS $^{\circledR} 22$. The data were analyzed using descriptive statistics, calculation of statistical parameters (the mean and standard deviation), student's t-test to determine the significant difference between groups.

\section{Results}

The distribution of the sample by age groups and sex for children with return consent form, and those who completed initial examinations is reported in table 1 . Figure 1 shows the mean combined dft/DFT by age and gender. Table 2 revealed the mean \pm standard deviation (SD) of combined $\mathrm{dft}$ and DFT by age groups and sex. There was no statistical significant difference between males and females for all age groups and for the total sample. The mean combined dft and DFT decreased with age with no significant differences. The only significant difference was between age group
6-8 year and $12-13$ year at $(\mathrm{p}<0.05)$. Table 3 shows a comparison between combined dft and DFT and SCI stratified by age groups. SCI for all age groups and for total sample were significantly higher than combined dft and DFT.

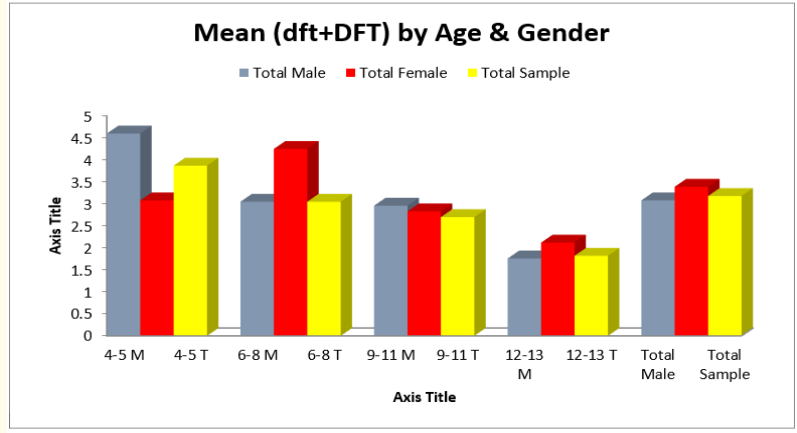

Figure 1

\begin{tabular}{|l|c|c|c|c|c|c|}
\hline \multirow{2}{*}{$\begin{array}{c}\text { Age group } \\
\text { (Years) }\end{array}$} & \multicolumn{2}{|c|}{ No. of children who returned consent form } & \multicolumn{3}{c|}{ No. of children who completed initial examination } \\
\cline { 2 - 7 } & Male & Female & Total & Male & Female & Total \\
\hline $4-5$ & 14 & 18 & 32 & 12 & 12 & 24 \\
\hline $6-8$ & 25 & 34 & 59 & 20 & 32 & 52 \\
\hline $9-11$ & 32 & 31 & 63 & 27 & 24 & 61 \\
\hline $12-13$ & 9 & 10 & 19 & 9 & 8 & 17 \\
\hline Total & 80 & 93 & 173 & 68 & 76 & 144 \\
\hline
\end{tabular}

Table 1: Distribution of the Sample by Age and Sex.

\begin{tabular}{|l|l|l|l|}
\hline \multicolumn{1}{|c|}{ Age Group (Years) } & \multicolumn{1}{c|}{ Sex } & \multicolumn{1}{c|}{ Mean } & \multicolumn{1}{c|}{ \pm SD } \\
\hline \multirow{2}{*}{$4-5$} & $\mathrm{M}$ & 4.66 & 4.792 \\
\cline { 2 - 4 } & $\mathrm{F}$ & 3.08 & 3.146 \\
\hline \multirow{2}{*}{ Total } & & 3.87 & 4.046 \\
\hline \multirow{2}{*}{ Total } & $\mathrm{M}$ & 3.05 & 3.252 \\
\hline \multirow{2}{*}{$9-11$} & $\mathrm{~F}$ & 4.25 & 3.636 \\
\hline Total & & $3.05^{*}$ & 3.510 \\
\hline \multirow{2}{*}{$12-13$} & $\mathrm{M}$ & 2.96 & 4.389 \\
\cline { 2 - 4 } & $\mathrm{F}$ & 2.83 & 4.360 \\
\hline Total & & 2.70 & 3.769 \\
\hline Total Males & $\mathrm{M}$ & 1.76 & 2.006 \\
\hline Total Females & $\mathrm{F}$ & 2.12 & 2.531 \\
\hline Total Sample & & $1.82^{*}$ & 2.214 \\
\hline
\end{tabular}

Table 2: Mean combined $(\mathrm{dft}+\mathrm{DFT}) \pm \mathrm{SD}$ by Age and Sex.

* Significant difference between total age group 6-8 year and $12-13$ year at $\mathrm{P}<0.05$

No significant differences between males and females.

\begin{tabular}{|l|l|l|l|l|}
\hline Age Group (Years) & Index & Mean & \pm SD & p-value \\
\hline \multirow{2}{*}{$4-5$} & $\mathrm{dft}+\mathrm{DFT}$ & 3.87 & 4.046 & \multirow{2}{*}{$<0.003$} \\
\cline { 2 - 4 } & sic & 8.75 & 2.314 & \\
\hline \multirow{2}{*}{$6-8$} & $\mathrm{dft}+\mathrm{DFT}$ & 3.05 & 3.510 & \multirow{2}{*}{$<0.000$} \\
\cline { 2 - 4 } & sic & 7.88 & 2.147 & \\
\hline \multirow{2}{*}{$9-11$} & $\mathrm{dft}+\mathrm{DFT}$ & 2.70 & 3.789 & \multirow{2}{*}{$<0.000$} \\
\cline { 2 - 4 } & sic & 7.05 & 3.578 & \\
\hline \multirow{3}{*}{$12-13$} & $\mathrm{dft}+\mathrm{DFT}$ & 1.82 & 2.214 & \multirow{2}{*}{$<0.035$} \\
\cline { 2 - 4 } & sic & 4.16 & 2.136 & \multirow{2}{*}{$<0.000$} \\
\hline \multirow{2}{*}{ Total } & $\mathrm{dft}+\mathrm{DFT}$ & 3.18 & 3.614 & \multicolumn{2}{|c}{} \\
\cline { 2 - 4 } & sic & 7.58 & 2.640 & \multicolumn{2}{|c}{} \\
\hline
\end{tabular}

Table 3: Comparison between combined ( $\mathrm{dft}+\mathrm{DFT})$ and SIC indices according to age group.

The number of caries free school children was 52 (36.1\%). Indicating the caries prevalence in this school was $63.9 \%$ (Table 4). The females tend to lower caries prevalence than males. The result shows that the ratio of decay teeth was $77 \%$ from the total teeth in dft and DFT. The percentage of caries prevalence in primary teeth 
Dental Caries Prevalence and Untreated Caries among Children in a School-Based Dental Health Program Using dft/DFT and Significant Caries Index (SCI)

was higher than in the permanent teeth and $32.6 \%$ of children had $81 \%$ of overall caries.

\begin{tabular}{|l|c|c|c|c|c|c|}
\hline \multirow{2}{*}{$\begin{array}{c}\text { Age Group } \\
\text { (Years) }\end{array}$} & \multicolumn{2}{|c|}{ Male } & \multicolumn{2}{c|}{ Female } & \multicolumn{2}{c|}{ Total } \\
\cline { 2 - 7 } & No. & \% & No. & \% & No. & \% \\
\hline $4-5$ & 5 & 41.6 & 4 & 33.3 & 9 & 37.5 \\
\hline $6-8$ & 7 & 35 & 8 & 25 & 15 & 28.8 \\
\hline $9-11$ & 12 & 35.7 & 10 & 32.2 & 22 & 36 \\
\hline $12-13$ & 4 & 44.4 & 2 & 25 & 6 & 32.3 \\
\hline Total & 28 & 41.1 & 24 & 31.5 & 52 & 36.1 \\
\hline
\end{tabular}

Table 4: Caries free for children by age and sex expressed as a number and percentage.

\section{Discussion}

The dmft/DMFT indices have been in use for more than 75 years and remain the most commonly index used to assessing dental caries prevalence. The mean $\mathrm{dmft} / \mathrm{DMFT}$ value is an average of all members of the sample, irrespective of the distribution of the severity of the dental caries within the sample. Many studies reported that mean dmft / DMFT values does not correctly reflect the skewed distribution, leaving high caries individuals undiscovered in the sample $[11,12]$. Therefore, Significant Caries Index (SCI) was proposed as a mean to highlight individuals with the high risk of dental caries in each sample.

The study revealed that the SCI was significantly higher than the mean dft/DFT in all age group and for total sample at $(\mathrm{P}<.0001)$. That indicates there is large subgroup (one third of the sample) with very high caries rate. The findings show that the mean score of SCI is about two and half times as high as the mean dft/DFT of the sample. In addition, the study shows that $32.6 \%$ of children have $81 \%$ of overall caries. Bratthal suggest a SCI of less than 3 in 12 year old children as a global oral health goal to be achieved by the year 2015 [13], our finding was higher than this goal. The SCI calculation gives a better picture for the high caries risk group in the population. Accordingly, we were able to draw attention to the group with highest caries experience. The findings of this study as well as others $[11,12,14]$ have indicated that several individuals suffer from a high prevalence of dental caries. For this reason, SCI index is a useful indicator and helps in targeting children with high caries risk in the community to establish a better preventive program and better access to oral health care. In order to improve the dental health and reduce dental care needs for high risk groups, there is a need for more school based dental sealant programs and early childhood fluoride varnish programs at three months period.

The study revealed that decay component of dft/DFT score had the greatest value $77 \%$. This means the presence of a high percentage of carious teeth that are untreated. Healthy people 2020 provides 10 -year national objectives for improving the overall health of Americans and seeks to eliminate health disparities [15]. The healthy people objectives for untreated decay are to reduce the proportion of 3 - 5 year olds with untreated decay in their primary teeth to $21 \%$ and for 6 - 9 year olds with untreated decay in their primary and permanent teeth to $26 \%$. When comparing our findings with other studies conducted in the US $[5,6]$ for similar age groups, the percentage of untreated teeth in this school were very high and exceeds the goals of healthy people 2020. In our study the percentage of children with untreated carious lesion were very high at about four times more than that reported in the NHANES data [16]. Almost all the caries were treated in these children in a short period of time. TFTMC is a viable solution to these children to provide them with full mouth treatment and to meet the goals of healthy people 2020 .

Caries prevalence in this sample was $63.9 \%$ for all groups. For age, 4 - 5 year caries prevalence was $62.5 \%$ and for age group 6 - 8 was $71.2 \%$. This prevalence is higher than that of the national mean of $33.3 \%$ and $54.4 \%$ respectively [16]. When compared with the data from Michigan study [6], it was also higher for age group of 6-8 year (58\%). The objectives of healthy people 2020 is to reduce the proportion of children aged 3 - 5 year with dental caries prevalence in their primary teeth to $30 \%$ and for 6 - 8 year in their primary and permanent teeth to $49 \%$. When comparing our findings to the objectives of healthy people 2020, it is found our percentages are very high specifically for age group 4 - 5 year.

The result shows that the percentage of caries in primary teeth was higher than in permanent teeth. This high proportion of decay in primary teeth reflects an unawareness of the importance of primary teeth or negative attitude of the parents toward dental treatment and in turn lack of cooperation of the children [17].

Dental caries is the most common childhood disease, and the majority of dental decay remains untreated due to inappropriate, unaffordable or unavailable oral care services [18]. Inability to 
access dental care, financial issues, parents' education, the value that parents place on primary teeth, dietary practice, the absence of fluoridated water, and other unidentified variables may be involved as explanatory factors for high caries prevalence and high percentage of untreated caries. Majority of the children in the sample were from low-income African American families. Inadequate access to dental care for children of low-income families may be largely due to lack of dental insurance. A study reported that individuals without dental insurance were 20 - 40\% more likely to have higher mean DMFT than those with dental insurance [19]. Other studies show that there is socioeconomic inequality in oral health in the American children. Individuals at lower end of the socioeconomic ladder have a higher burden of dental disease compared to those who are socioeconomic better off $[20,21]$.

\section{Conclusion}

The SCI is designed to focus attention to high caries risk group and is useful indicator and helps in targeting the preventive program for this group. The high caries prevalence together with the low level of dental care is very important to establish a better preventive dental program and focus on a high-risk group. A lower socioeconomic status in this group may strongly influence the prevalence of dental caries and high percentage of untreated caries. UDMSOD TFTMC is a viable solution to these children, who for variety of reasons have been unable to access adequate dental care. There is a need to further study the children in the TFTMC program to evaluate DFT, $\mathrm{dft}$ and SCI and the need for dental care.

Why this paper is important to pediatric dentist?

- High risk children still missing access to dental care.

- $\quad$ To apply prevention plan we have to target the pediatric population.

- Using the appropriate tool in our data collection will help in delivering the appropriate care.

\section{Bibliography}

1. FDI World Dental Federation: A report by FDI World Dental Federation. World Oral Health Day (2014).

2. US Department of Health and Human services: Oral Health in America. A report of the Surgeon General.
3. National Center for Health Statistics. "Current estimates from the National Health Interview Survey, 1996". Vital and Health Statistics Series 10200 (1996): 1-203.

4. Dye B., et al. "Oral health disparities as determined by selected healthy people 2020, Oral health objectives for the United States, 2009-2010". NCHS Data Brief 104 (2012): 1-8.

5. Dye B., et al. "Dental caries and sealant prevalence in children and adolescents in the United States 2011-2012". NCHS Data Brief 191 (2015): 1-8.

6. Michigan Department of Community Health: Burden of Oral Disease in Michigan (2013).

7. Neurath C. "Tooth decay trends for 12 year olds in nonfluoradated and fluoradated countries". Fluoride 38.4 (2005): 324325.

8. Zander A., et al. "Risk factors for dental caries in small rural and regional Australian communities". Rural Remote Health 13.3 (2013): 2492.

9. Lagerweij M and Van Loveren C. "Declining caries trends: Are we satisfied”. Current Oral Health Reports 2.4 (2015): 212-217.

10. Bratthall D. "Introducing the significant caries index together with a proposal for a new global oral health goal for 12 year olds". International Dental Journal 50.6 (2000): 378-384.

11. Ditmyer M., et al. "Inequalities of caries experience in Nevada youth expressed by DMFT index VS. Significant caries index (SCI) over time". BMC Oral Health 11.1 (2011): 12-21.

12. Gasgoos S., et al. "evaluation of dental caries prevalence among children in Mosul City center using significant caries index". AlRafidain Dental Journal 12.19 (2012): 191-197.

13. Bratthal D. "Introducing the significant caries index together with proposal for a new global oral health for 12 year olds". International Dental Journal 50.6 (2000): 378-384.

14. Jokic N., et al. "dental caries experience in Croatian school children in Primorsk-Goranska County". Central European Journal of Public Health 21.1 (2013): 39-42. 
Dental Caries Prevalence and Untreated Caries among Children in a School-Based Dental Health Program Using dft/DFT and Significant Caries Index (SCI)

15. Office of Disease Prevention and Public Health Promotion (ODPHP): Healthy people (2020).

16. National Health and Nutrition Examination Survey (NHANES): centers for Disease Control and Prevention, National Center for Health statistics (CDC/NCHS); year 1999-2004.

17. Baghdady V and Ghose L. "Dental caries prevalence in school children of Baghdad province, Iraq". Community Dentistry and Oral Epidemiology 10.3 (1982): 148-151.

18. Oral Health: a report by FDI World Dental Federation: World oral health Day (2014).

19. Mouradian W., et al. "Disparties in children's oral health and access to dental care". The Journal of the American Medical Association 284.20 (2000): 2625-2631.

20. Copurro D., et al. "trends in income-related inequality in untreated caries among children in the United States. Findings from NHANES I, NHANES III and NHANES 1999-2004". Community Dentistry and Oral Epidemiology 43.6 (2015): 500-510.

21. Dye B., et al. "Trends in paediatric dental caries by poverty status in the United States, 1988-1994 and 1999- 2004". International Journal of Paediatric Dentistry 20.2 (2010): 132-114.

\section{Volume 3 Issue 10 October 2019}

(C) All rights are reserved by Tarik Shounia., et al. 